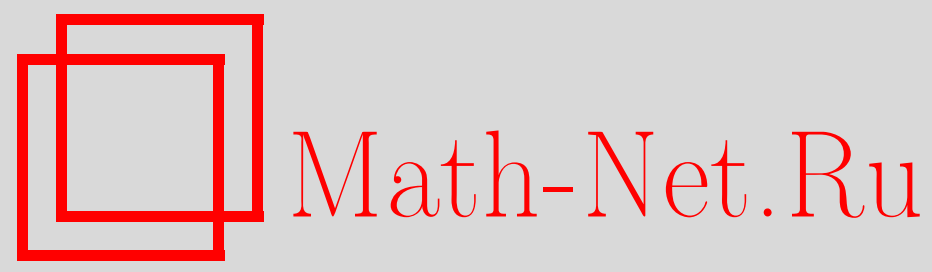

Г. В. Коваль, Асимптотика по числу элементов статистического ансамбля в уравнениях квантовой термодинамики В.П. Маслова, Матем. заметки, 2003, том 73, выпуск 4, 631-633

DOI: https://doi.org/10.4213/mzm627

Использование Общероссийского математического портала Math-Net.Ru подразумевает, что вы прочитали и согласны с пользовательским соглашением http://www.mathnet.ru/rus/agreement

Параметры загрузки:

IP : 54.162 .127 .20

26 апреля 2023 г., 15:44:20

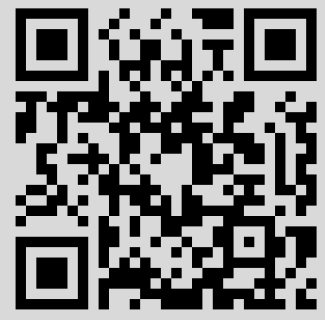




\section{АСИМПТОТИКА ПО ЧИСЛУ ЭЛЕМЕНТОВ СТАТИСТИЧЕСКОГО АНСАМБЛЯ В УРАВНЕНИЯХ КВАНТОВОЙ ТЕРМОДИНАМИКИ В.П. МАСЛОВА}

\section{Г.В. Коваль}

В данной работе на примере простой модели показано, что при построении асимптотик решений следует рассматривать не только периодические траектории возникающей классической Гамильтоновой системы уравнений, но и траектории, заполняющие инвариантные торы. В рассмотренной модельной задаче найдены инвариантные торы и показано, что отвечающий им канонический оператор является асимптотическим решением задачи.

В работах В. П. Маслова [1], [2], посвященных квантованию термодинамики, возникла следующая задача на собственные значения:

$$
\begin{gathered}
\sum_{\alpha=1}^{m}\left(\lambda_{\alpha} \hat{n}_{\alpha}+\theta \hat{n}_{\alpha} \ln \left(\frac{\hat{n}_{\alpha}}{k}\right)\right) \Phi=f \Phi, \quad \Phi \neq 0, \\
\sum_{\alpha=1}^{m} \hat{n}_{\alpha} \Phi=k \Phi,
\end{gathered}
$$

где $0<\theta$ - действительньй параметр, $0 \leqslant k$ - заданное целое число, $\Phi$ - вектор бозонного фоковского пространства $\mathscr{F}_{B}[3]$, порожденного вакуумным вектором $\Phi_{0}$ и операторами рождения $\hat{b}_{\alpha}^{+}$и уничтожения $\hat{b}_{\alpha}^{-}, \alpha=1, \ldots, m$, удовлетворяющими соотношениям

$$
\left[\hat{b}_{\alpha}^{-}, \hat{b}_{\alpha^{\prime}}^{+}\right]=\delta_{\alpha \alpha^{\prime}}, \quad\left[\hat{b}_{\alpha}^{ \pm}, \hat{b}_{\alpha^{\prime}}^{ \pm}\right]=0, \quad \hat{b}_{\alpha}^{-} \Phi_{0}=0 ;
$$

кроме того, в формулах (1), (2) $\hat{n}_{\alpha}-$ следуюшие операторы в пространстве $\mathscr{F}_{B}: \hat{n}_{\alpha}=\hat{b}_{\alpha}^{+} \hat{b}_{\alpha}^{-}$. Собственные значения $f$ согласно [1], [2] являются собственными значениями свободной энергии при температуре $\theta$ ансамбля из $k$ одинаковых систем, каждая из которых имеет дискретный спектр энергии $\lambda_{\alpha}, \alpha=1, \ldots, m$. Решения уравнения (1) с дополнительным условием (2) хорошо известны, они имеют вид

$$
f_{\{n\}}=\sum_{\alpha=1}^{m}\left(\lambda_{\alpha} n_{\alpha}+\theta n_{\alpha} \ln \left(\frac{n_{\alpha}}{k}\right)\right), \quad \Phi_{\{n\}}=\prod_{\alpha=1}^{m} \frac{\left(\hat{b}_{\alpha}^{+}\right)^{n_{\alpha}}}{\sqrt{n_{\alpha} !}} \Phi_{0},
$$

где $\{n\}$ обозначает набор целых чисел $0 \leqslant n_{\alpha}, \alpha=1, \ldots, m$, таких что

$$
\sum_{\alpha=1}^{m} n_{\alpha}=k \text {. }
$$

В пределе при $k \rightarrow \infty$ согласно [1], [2] из множества значений (3) можно выделить серии собственных значений (понятие серии см. в [4]). Каждая такая серия определяется набором целых чисел $J$, где $J$ - подмножество множества $\{1, \ldots, m\}$, а главный член асимптотики минимального значения $f$, принадлежащего серии, имеет вид

$$
F_{J}=-k \theta \ln \left(\sum_{\alpha \in J} e^{-\lambda_{\alpha} / \theta}\right)
$$

где через $\sum_{\alpha \in J}$ обозначена сумма по всем числам $\alpha$, принадлежащим множеству $J$.

Работа выполнена при финансовой поддержке Российского фонда фундаментальных исследований, грант № 02-01-1062. 
Заметим теперь, что в уравнении (1) и условии (2) параметр $k$ стоит таким образом, что для нахождения асимптотики решений при $k \rightarrow \infty$ применимы методы, развитые в [5]-[7]. При этом сериям, полученным в [1], [2], соответствуют периодические решения возникающей в квазиклассическом приближении системы уравнений Гамильтона. Рассмотрим представление [8], в котором элементам фоковского пространства $\mathscr{F}_{B}$ соответствуют функции $\Psi\left(q_{1}, \ldots, q_{m}\right) \in L_{2}\left(\mathbb{R}^{m}\right)$, а операторы $\hat{b}_{\alpha}^{+}$и $\hat{b}_{\alpha}^{-}$имеют вид

$$
\hat{b}_{\alpha}^{+}=\sqrt{\frac{k}{2}}\left(q_{\alpha}-\frac{1}{k} \frac{\partial}{\partial q_{\alpha}}\right), \quad \hat{b}_{\alpha}^{-}=\sqrt{\frac{k}{2}}\left(q_{\alpha}+\frac{1}{k} \frac{\partial}{\partial q_{\alpha}}\right) .
$$

В этом представлении уравнение (1) и дополнительное условие (2) принимают вид

$$
\begin{aligned}
& \sum_{\alpha=1}^{m}\left(\lambda_{\alpha} \hat{n}_{\alpha}+\theta \llbracket-\frac{1}{2 k^{2}} \frac{\partial^{2}}{\partial q_{\alpha}^{2}}+\frac{q_{\alpha}^{2}}{2}-\frac{1}{2 k} \rrbracket \ln \left(\llbracket-\frac{1}{2 k^{2}} \frac{\partial^{2}}{\partial q_{\alpha}^{2}}+\frac{q_{\alpha}^{2}}{2}-\frac{1}{2 k} \rrbracket\right)\right) \Psi\left(q_{1}, \ldots, q_{m}\right) \\
& \quad=f \Psi\left(q_{1}, \ldots, q_{m}\right), \\
& \sum_{\alpha=1}^{m}\left(-\frac{1}{2 k^{2}} \frac{\partial^{2}}{\partial q_{\alpha}^{2}}+\frac{q_{\alpha}^{2}}{2}-\frac{1}{2 k}\right) \Psi\left(q_{1}, \ldots, q_{m}\right)=k \Psi\left(q_{1}, \ldots, q_{m}\right),
\end{aligned}
$$

где 【· 』обозначает автономные скобки [5]-[7]. Задача о нахождении асимптотики решений уравнения (5) при $k \rightarrow \infty$ является задачей о построении квазиклассической асимптотики [5]-[7], роль постоянной Планка $\hbar$ играет параметр $1 / k$, а оператору, действующему на функцию $\Phi$ в левой части уравнения (5), соответствует функция Гамильтона

$$
H(q, p)=\sum_{\alpha=1}^{m}\left(\lambda_{\alpha}+\theta \ln \left(\frac{q_{\alpha}^{2}+p_{\alpha}^{2}}{2}\right)\right) \frac{q_{\alpha}^{2}+p_{\alpha}^{2}}{2} .
$$

Система уравнений Гамильтона, отвечающая функции (7), имеет вид

$\dot{q}_{\alpha}=\left(\lambda_{\alpha}+\theta+\theta \ln \left(\frac{q_{\alpha}^{2}+p_{\alpha}^{2}}{2}\right)\right) p_{\alpha}, \quad \dot{p}_{\alpha}=-\left(\lambda_{\alpha}+\theta+\theta \ln \left(\frac{q_{\alpha}^{2}+p_{\alpha}^{2}}{2}\right)\right) q_{\alpha}, \quad \alpha=1, \ldots, m$.

Система уравнений (8) является системой с разделяющимися переменными, ее решения имеют вид

$$
q_{\alpha}(t)=\sqrt{2 I_{\alpha}} \sin \left(\Omega_{\alpha} t+\varphi_{\alpha}\right), \quad p_{\alpha}(t)=\sqrt{2 I_{\alpha}} \cos \left(\Omega_{\alpha} t+\varphi_{\alpha}\right), \quad \alpha=1, \ldots, m,
$$

где $I_{\alpha}, \varphi_{\alpha}$ определяются значениями $q_{\alpha}$ и $p_{\alpha}$ в момент времени $t=0$, а $\Omega_{\alpha}$, если $I_{\alpha} \neq 0$, выражаются следующим образом:

$$
\Omega_{\alpha}=\lambda_{\alpha}+\theta+\theta \ln \left(I_{\alpha}\right) .
$$

В [7] показано, что периодическим решениям системы (8) вида (9) (т.е. решениям, у которых все $\Omega_{\alpha}$ вида (10) равны), удовлетворяющим условию квантования [6], [7] $\sum_{\alpha=1}^{m} I_{\alpha}=(n+1 / 2) / k$, где $n-$ целое число, соответствуют серии решений уравнения (1). Дополнительное условие (6) накладывает ограничение на рассматриваемые периодические траектории: $n=k$. Легко убедиться, что все периодические решения системы (7) задаются формулой (9), в которой $\varphi_{\alpha}$ произвольные, а $I_{\alpha}$ имеют вид

$$
I_{\alpha}=\exp \left(\frac{\Omega-\lambda}{\theta}-1\right), \quad \text { если } \alpha \in J, \quad \text { и } I_{\alpha}=0, \quad \text { если } \alpha \notin J,
$$

где $J$ - подмножество множества $\{1, \ldots, m\}$, а $\Omega$ - частота периодической траектории. Условие квантования выделяет для каждого $J$ одну периодическую траекторию. И этой периодической траектории соответствует серия решений уравнения (5) с дополнительным условием (6), которая отвечает набору целых чисел $J$, а минимальное собственное значение $f$ которой имеет вид (4).

Заметим теперь, что в общем случае, когда в формуле (9) числа $\Omega_{\alpha}$ при разных $\alpha=1, \ldots, m$ несоизмеримы, траектория в фазовом пространстве $\mathbb{R}^{2 m}$, задаваемая этой формулой, не является 
периодической, но она всюду плотно заполняет $m$-мерный тор, параметрическое уравнение которого имеет вид

$$
q_{\alpha}=\sqrt{2 I_{\alpha}} \sin \left(\phi_{\alpha}\right), \quad p_{\alpha}=\sqrt{2 I_{\alpha}} \cos \left(\phi_{\alpha}\right), \quad \alpha=1, \ldots, m,
$$

где $\phi_{\alpha} \in[0,2 \pi)$. Если же $\Omega_{\alpha}$ соизмеримы, например все равны $\Omega$, то имеется бесконечное множество траекторий, объединение которых равно тору (12). Например, любому набору $\varphi_{\alpha} \in[0,2 \pi)$, $\alpha=1, \ldots, m$, формула (9) сопоставляет свою периодическую траекторию и легко убедиться, что объединение всех таких траекторий есть множество, задаваемое формулой (12). Тор (12) является лагранжевым многообразием, инвариантным относительно гамильтоновой системы (8) [6]. В монографии [6] рассмотрено это лагранжево многообразие и приведены условия квантования, выполнение которых позволяет построить на нем канонический оператор. Условия квантования тора (12) согласно [6] есть $I_{\alpha}=\left(n_{\alpha}+1 / 2\right) / k$, где $n_{\alpha}$ - целые числа. Канонический оператор, отвечающий такому квантованному тору удовлетворяет условию (6), если для чисел $n_{\alpha}$ справедливо равенство $\sum_{\alpha=1}^{m} n_{\alpha}=n$, кроме того этот канонический оператор является асимпотическим решением уравнения (5), а соответствуюшее значение $f$ совпадает с точным решением, которое имеет вид (3).

Таким образом, в данной статье установлено соответствие между решениями системы уравнений Гамилтона (8), покрывающими инвариантный тор, и точными решениями уравнения (1) с дополнительным условием (2). При этом оказалось, что построенная в [1], [2], [5] асимптотика отвечает случаю, когда инвариантный тор вырождается - разбивается на непересекающиеся периодические траектории гамильтоновой системы. Поэтому представляет значительный интерес нахождение более сложных, чем периодические траектории, инвариантных многообразий гамильтоновой системы и соответствующих асимптотик в более сложных, чем рассмотренная в данной статье задачах.

\section{СПИСОК ЦИТИРОВАННОЙ ЛИТЕРАТУРЫ}

1. Маслов В.П. // Матем. заметки. 2002 (в печати). 2. Маслов В. П. // ТМФ. 2002 (в печати). 3. Березин Ф.А. Метод вторичного квантования. М.: Наука, 1987. 4. Маслов В. П. Квантование термодинамики и ультравторичное квантование. Ижевск: НИЦ "Регулярная и хаотическая динамика", УРСС, 2001. 5. Маслов В. П., Шведов О. Ю. Метод комплексного ростка в многочастичных задачах и задачах квантовой теории поля. М.: УРСС, 2000. 6. Маслов В. П., Федорюк М. В. Квазиклассическое приближение для уравнений квантовой механики. М.: Наука, 1976. 7. Маслов В. П. Комплексный метод ВКБ в нелинейных уравнениях. М.: Наука, 1977. 8. Березин Ф. А., Шубин М. А. Уравнение Шрёдингера. М.: Изд-во Московского ун-та, 1983. 